\title{
ORTHOGONALLY ADDITIVE POLYNOMIALS ON C*-ALGEBRAS
}

\author{
CARLOS PALAZUELOS, ANTONIO M. PERALTA, AND IGNACIO VILLANUEVA
}

\begin{abstract}
Let $A$ be a $C^{*}$-algebra which has no quotient isomorphic to $M_{2}(\mathbb{C})$. We show that for every orthogonally additive scalar $n$ homogeneous polynomials $P$ on $A$ such that $P$ is Strong* continuous on the closed unit ball of $A$, there exists $\varphi$ in $A^{*}$ satisfying that $P(x)=\varphi\left(x^{n}\right)$, for each element $x$ in $A$. The vector valued analogue follows as a corollary.
\end{abstract}

\section{INTRODUCTION}

Let $A$ be a $\mathrm{C}^{*}$-algebra. Two elements $x, y$ in $A$ are said to be orthogonal if $x y=y x=0$. A mapping $\Phi$ from $A$ to a Banach space is said to be orthogonally additive if for every pair of mutually orthogonal elements $x, y$ in $A$ we have $\Phi(x+y)=\Phi(x)+\Phi(y)$.

In the case of a Banach lattice $L$, a function $\Phi$ from $L$ to a Banach space is said to be orthogonally additive if $\Phi(x+y)=\Phi(x)+\Phi(y)$, whenever $x$ and $y$ are orthogonal.

By an $X$-valued $n$-homogeneous polynomial on a Banach space $E$ we mean a continuous $X$-valued mapping $P$ on $E$ for which there exists a continuous $n$-linear operator $T: E \times \cdots \times E \longrightarrow X$ satisfying $P(x)=T(x, \ldots, x)$, for every $x$ in $X$.

Orthogonally additive polynomials on Banach lattices have been studied and described by several authors (see for instance $[2,7,8,9,16,20]$ ).

When, by the Gelfand-Naimark theorem, the $C(K)$ spaces are regarded as unital abelian $\mathrm{C}^{*}$-algebras, the main result in [16] asserts that for each orthogonally additive $n$-homogeneous polynomial $P: C(K) \rightarrow \mathbb{C}$, there exists a Borel regular measure $\mu$ on $K$ such that $P(f)=\int_{K} f^{n} d \mu,(f \in C(K))$. In other words, for each $P$ as above, there exists $\phi$ in $C(K)^{*}$ satisfying that $P(f)=\phi\left(f^{n}\right),(f \in C(K))$.

It seems natural to ask whether the above description remains true for orthogonally additive homogeneous polynomials on a general $\mathrm{C}^{*}$-algebra. This is the main goal treated in this note. It is not hard to see that for each $\phi$ in the dual space of a $\mathrm{C}^{*}$-algebra $A$, the law $x \mapsto P_{\phi}(x):=\phi\left(x^{n}\right)$

First and third author partially supported by I+D MCYT project no MTM2005-00082 and second author partially supported by I+D MCYT project no. MTM2005-02541, and Junta de Andalucía grants FQM0199 and FQM1215. 
defines an $n$-homogeneous polynomial $P_{\phi}$ on $A$. Since, for each pair of mutually orthogonal elements $x, y \in A$, we have $(x+y)^{n}=x^{n}+y^{n}$, it follows that $P_{\phi}$ is orthogonally additive. We shall check in $\S 3$, that the above polynomial $P_{\phi}$ also has the following topological property: $P_{\phi}$ is $S^{*}\left(A, A^{*}\right)$ to-norm continuous on the closed unit ball of $A$ (see definitions bellow). Section two provides a study of those multilinear operators on the cartesian product of $\mathrm{C}^{*}$-algebras and Banach spaces which are jointly Strong*-to-norm continuous on the closed unit balls.

Section three deals with the study of orthogonally additive homogeneous polynomials on a $\mathrm{C}^{*}$-algebra. When considering (non necessarily unital) abelian $\mathrm{C}^{*}$-algebras, the description provided in [16] can be adapted word by word, to show that for every $M \in \mathbb{R}^{+}$, every locally compact space $L \subset(0, M]$, such that $L \cup\{0\}$ is compact, and every orthogonally additive homogeneous polynomials $P$ on $C_{0}(L)$, there exists a functional $\phi \in C_{0}(L)^{*}$ satisfying that $P(f)=\phi\left(f^{n}\right)$ (Proposition 12). This result guarantees every orthogonally additive homogeneous polynomials on a $\mathrm{C}^{*}$-algebra can be "locally" good-described on the subalgebra generated by a self-adjoint element. The result that allows us to extend this "local" description to a global description will follow from the solution of the Mackey-Gleason problem given by L. J. Bunce and J. D. M. Wright in $[4,5]$ and [6]. More concretely, Theorem 3.1 in [6] gives us the key tool used in Proposition 13 to prove linearity for quasi-linear mappings on a $\mathrm{C}^{*}$-algebra.

Finally, in our main result (Theorem 16) we establish the following description: Let $A$ be a $\mathrm{C}^{*}$-algebra which has no quotient isomorphic to $M_{2}(\mathbb{C})$, and let $P$ be an orthogonally additive $n$-homogeneous polynomials $P$ on $A$ which is Strong* continuous on the closed unit ball of $A$, then there exists $\varphi$ in $A^{*}$ satisfying that $P(x)=\varphi\left(x^{n}\right)$, for each element $x$ in $A$. The vector valued case follows easily.

Given Banach spaces $X$ and $Y, L(X, Y)$ will denote the space of all bounded linear mappings from $X$ to $Y$. Throughout the paper the word "operator" will always mean bounded linear operator. We shall write $L(X)$ instead of $L(X, X)$. The symbol $\mathcal{P}^{n}(X, Y)$ will stand for the Banach space of all n-homogeneous polynomials from $X$ to $Y$. When $Y=\mathbb{K}$ we will omit it. The closed unit ball of a Banach space $X$ is denoted by $B_{X}$ while $X^{*}$ will stand for its dual space.

Let $X_{1}, \ldots, X_{n}$, and $X$ be Banach spaces and $T: X_{1} \times \cdots \times X_{n} \rightarrow X$ be a $n$-linear operator. Let $\pi:\{1, \ldots, n\} \rightarrow\{1, \ldots, n\}$ be a permutation. There is a unique $n$-linear extension $A B(T)_{\pi}: X_{1}^{* *} \times \cdots \times X_{n}^{* *} \rightarrow X^{* *}$ verifying, for every $z_{i} \in X_{i}^{* *}$ and every net $\left(x_{\alpha_{i}}^{i} \in X_{i}(1 \leq i \leq n)\right.$

$$
A B(T)_{\pi}\left(z_{1}, \ldots, z_{k}\right)=\text { weak }^{*}-\lim _{\alpha_{\pi(1)}} \cdots \text { weak }^{*}-\lim _{\alpha_{\pi(k)}} T\left(x_{\alpha_{1}}^{1}, \ldots, x_{\alpha_{n}}^{n}\right) .
$$

Moreover, $A B(T)_{\pi}$ is bounded and has the same norm as $T$. The extensions $A B(T)_{\pi}$ are the same as the extensions constructed by Aron and 
Berner for polynomials in [1], and we call them the Aron-Berner extensions of $T$ (see also [15, Proposition 3.1]).

Let $W$ be a von Neumann algebra and let $\phi$ be a positive functional in $W_{*}$, the law $z \mapsto \phi\left(z z^{*}\right)$ defines a seminorm on $W$. The Strong topology (respectively, the Strong* topology) on $W$ is the topology generated by all the seminorms $\phi\left(z z^{*}\right)$ (respectively, $\left.\phi\left(\frac{z^{*} z+z z^{*}}{2}\right)\right)$ ) where $\phi$ runs on all positive functionals in $W_{*}$. The Strong* topology on $W$ is usually denoted by $S^{*}\left(W, W_{*}\right)$. Given a $\mathrm{C}^{*}$-algebra $A$, the Strong* topology on $A\left(S^{*}\left(A, A^{*}\right)\right)$ is the topology $\left.S^{*}\left(A^{* *}, A^{*}\right)\right|_{A}$. In $[15, \S 2]$ the reader can find several interesting characterizations of the Strong* topology on Banach spaces.

\section{Joint Strong*-Continuity of Multilinear maps}

The following characterization of the joint Right-to-norm continuity for $T$ is borrowed from [15, Proposition 3.20]: Let $X_{1}, \ldots, X_{n}$ and $X$ be Banach spaces and let $T: X_{1} \times \cdots \times X_{n} \rightarrow X$ be a multilinear operator. Consider the following statements:

a) $T$ is jointly Right-to-norm continuous.

b) There exists a positive constant $M$ (depending only on $T$ ), reflexive Banach spaces $R_{1}, \ldots, R_{n}$ and bounded linear operators $T_{i}: X_{i} \rightarrow R_{i}$ satisfying, for each $x_{i}$ in $X_{i}$,

$$
\left\|T\left(x_{1}, \ldots, x_{n}\right)\right\| \leq M\left\|\left|x_{1}\right|\right\|_{T_{1}} \cdots\left\||| x_{n} \mid\right\|_{T_{n}} .
$$

c) $T$ is jointly Right-to-norm continuous on bounded subsets.

Then the following implications hold a) $\Rightarrow \mathrm{b}) \Rightarrow \mathrm{c}$ ).

We recall that for each bounded operator $T$ between two Banach spaces $X$ and $Y$, the symbol $\||\cdot|\|_{T}$ will denote the seminorm on $X$ defined by $x \mapsto\||x|\|_{T}:=\|T(x)\|$.

The above result remains true when we replace the Right topology with the $S^{*}\left(X_{i}, X_{i}^{*}\right)$ topology and the reflexive spaces are assumed to be Hilbert spaces (see $[15, \S 3]$ ).

Another interesting question is whether the joint Strong*-to-norm continuity on the cartesian product of the closed unit balls could be also characterised. In the linear case, [13, Theorem 2.4] establishes the following:

Proposition 1. [13] Let $X$ and $Y$ be Banach spaces and $T: X \rightarrow Y$ a linear operator. The following are equivalent:

a) $\left.T\right|_{B_{X}}: B_{X} \rightarrow Y$ is Right $\left.\right|_{B_{X}}$-to-norm continuous;

b) There exist a bounded linear operator $S$ from $X$ into a reflexive Banach space and a mapping $N:(0,+\infty) \rightarrow(0,+\infty)$ satisfying that

$$
\|T(x)\| \leq N(\varepsilon)\||x|\|_{S}+\varepsilon\|x\|,
$$

for all $x \in X, \varepsilon>0$.

In $[14,13]$, it was shown that conditions $a)$ and $b$ ) in the above results are also equivalent to: 
a)' $T$ is weakly compact;

b)' $T$ is Right-to-norm continuous.

Using the polarization formula for symmetric multilinear operators [11, p. 6] we easily get the following:

Lemma 2. Let $X$ and $Y$ be a Banach spaces, $P \in \mathcal{P}^{n}(X, Y)$ an n-homogeneous polynomial and $T: X \times \ldots \times X \rightarrow Y$ the symmetric multilinear operator associated to it. Then $\left.T\right|_{B_{X} \times \ldots \times B_{X}}$ is jointly $S^{*}\left(X, X^{*}\right)$-to-norm continuous if and only if $\left.P\right|_{B_{X}}$ is $S^{*}\left(X, X^{*}\right)$-to-norm continuous.

Lemma 3. Let $X_{1}, \ldots, X_{n}$ and $X$ be Banach spaces and let

$$
S: X_{1} \times \cdots \times X_{n} \rightarrow X
$$

be a multilinear operator. Suppose that

$$
\left.S\right|_{B_{X_{1}} \times \ldots \times B_{X_{n}}}: B_{X_{1}} \times \ldots \times B_{X_{n}} \rightarrow X
$$

is jointly Strong*-to-norm continuous, then there are Hilbert spaces $H_{1}$, $H_{2}, \ldots, H_{n}$ and bounded linear operators $S_{i}: X_{i} \rightarrow H_{i}$ such that

$$
\left\|S\left(x_{1}, \ldots, x_{n}\right)\right\| \leq \prod_{i=1}^{n}\left(\left\|\left|x_{i}\right|\right\|_{S_{i}}+\left\|x_{i}\right\|\right),
$$

for every $x_{i} \in X_{i}$.

Proof. According to our hypothesis, the set

$$
\mathcal{O}:=\left\{\left(x_{1}, \ldots, x_{n}\right) \in B_{X_{1}} \times \ldots \times B_{X_{n}}:\left\|T\left(x_{1}, \ldots, x_{n}\right)\right\| \leq 1\right\}
$$

is a Strong*-neighborhood of 0 in $B_{X_{1}} \times \ldots \times B_{X_{n}}$. Thus, by the definition of the Strong* topology, for each $i=1, \ldots n$, there exist a positive constant $\delta$, Hilbert spaces $H_{1}^{i}, \ldots, H_{p_{i}}^{i}$ and bounded linear operators $T_{j}^{i}: X_{i} \rightarrow H_{j}^{i}\left(1 \leq j \leq p_{i}\right)$, such that

$$
\mathcal{O} \supseteq \mathcal{O}^{\prime}:=\left\{\left(x_{1}, \ldots, x_{n}\right) \in B_{X_{1}} \times \ldots \times B_{X_{n}}:\left\|\left|x_{i}\right|\right\|_{T_{j}^{i}} \leq \delta, \forall 1 \leq j \leq p_{i}\right\} .
$$

We denote

$$
H_{i}:=\bigoplus_{1 \leq j \leq p_{i}}^{\ell_{2}} H_{j}^{i}
$$

and $S_{i}: X_{i} \rightarrow H_{i}$ the bounded linear operator given by $S_{i}\left(x_{i}\right):=\left(\delta^{-1} T_{j}^{i}(x)\right)$. Clearly, for each $i, H_{i}$ is a Hilbert space.

For each $\left(x_{1}, \ldots, x_{n}\right) \in X_{1}, \ldots, X_{n}$, with $x_{i} \neq 0(1 \leq i \leq n)$, the element

$$
\left(\frac{1}{\left\|S_{1}\left(x_{1}\right)\right\|+\left\|x_{1}\right\|} x_{1}, \ldots, \frac{1}{\left\|S_{n}\left(x_{n}\right)\right\|+\left\|x_{n}\right\|} x_{n}\right)
$$

belongs to $\mathcal{O}^{\prime} \subseteq \mathcal{O}$, and hence

$$
\left\|T\left(\frac{1}{\left\|S_{1}\left(x_{1}\right)\right\|+\left\|x_{1}\right\|} x_{1}, \ldots, \frac{1}{\left\|S_{n}\left(x_{n}\right)\right\|+\left\|x_{n}\right\|} x_{n}\right)\right\| \leq 1
$$


which implies that

$$
\left\|T\left(x_{1}, \ldots, x_{n}\right)\right\| \leq \prod_{i=1}^{n}\left(\left\|\left|x_{i}\right|\right\|_{S_{i}}+\left\|x_{i}\right\|\right) .
$$

When any $x_{i}=0$, the above inequality is trivial.

We can now prove:

Proposition 4. Let $X_{1}, \ldots, X_{n}$ and $X$ be Banach spaces and let

$$
T: X_{1} \times \cdots \times X_{n} \rightarrow X
$$

be a multilinear operator. Then the following are equivalent:

a) $\left.T\right|_{B_{X_{1}} \times \ldots \times B_{X_{n}}}: B_{X_{1}} \times \ldots \times B_{X_{n}} \rightarrow X$ is jointly Strong*-to-norm continuous.

b) There exist mappings $N_{i}:(0,+\infty) \rightarrow(0,+\infty)$ (depending only on $T)$, Hilbert spaces $H_{1}, \ldots, H_{n}$ and bounded linear operators $T_{i}$ : $X_{i} \rightarrow R_{i}$ satisfying that

$$
\left\|T\left(x_{1}, \ldots, x_{n}\right)\right\| \leq \prod_{i=1}^{n}\left(N_{i}(\varepsilon)\left\|\left|x_{i}\right|\right\|_{T_{i}}+\varepsilon\left\|x_{i}\right\|\right),
$$

for each $x_{i}$ in $X_{i}$ and $\varepsilon>0$.

Proof. $a) \Rightarrow b$ ) For each natural $m$, the mapping $m T$ is is jointly Strong*-tonorm continuous on $B_{X_{1}} \times \ldots \times B_{X_{n}}$. Thus, by Lemma 3 , there are Hilbert spaces $H_{m}^{i}(1 \leq i \leq n)$ and bounded linear operators $S_{i, m}: X_{i} \rightarrow H_{n}^{i}$ satisfying that

$$
\left\|m T\left(x_{1}, \ldots, x_{n}\right)\right\| \leq \prod_{i=1}^{n}\left(\left\|\left|x_{i}\right|\right\|_{S_{i, m}}+\left\|x_{i}\right\|\right),
$$

for all $\left(x_{1}, \ldots, x_{n}\right)$. We may assume $S_{i, m} \neq 0$, for all $m \in \mathbb{N}, 1 \leq i \leq n$. Define

$$
H_{i}:=\bigoplus_{m \in \mathbb{N}}^{\ell_{2}} H_{m}^{i}
$$

and

$$
S_{i}: X_{i} \rightarrow H_{i}
$$

the bounded linear operator given by

$$
S_{i}\left(x_{i}\right):=\left(\frac{1}{m\left\|S_{i, m}\right\|} S_{i, m}\left(x_{i}\right)\right),
$$

and $N_{i}:(0,+\infty) \rightarrow(0,+\infty)$ by $N_{i}(\varepsilon):=\left\|S_{i, m(\varepsilon)}\right\|$, where

$$
m(\varepsilon)=\inf \left\{m \in \mathbb{N}: \frac{1}{\sqrt[n]{m}}<\varepsilon\right\}
$$


Finally, given $\left(x_{1}, \ldots, x_{n}\right) \in X_{1} \times \ldots \times X_{n}$ we have

$$
\begin{gathered}
\left\|m(\varepsilon) T\left(x_{1}, \ldots, x_{n}\right)\right\| \leq \prod_{i=1}^{n}\left(\left\|S_{i, m(\varepsilon)}\left(x_{i}\right)\right\|+\left\|x_{i}\right\|\right) \\
\left\|T\left(x_{1}, \ldots, x_{n}\right)\right\| \leq \prod_{i=1}^{n}\left(\frac{1}{\sqrt[n]{m(\varepsilon)}}\left\|S_{i, m(\varepsilon)}\left(x_{i}\right)\right\|+\frac{1}{\sqrt[n]{m(\varepsilon)}}\left\|x_{i}\right\|\right) \\
\left\|T\left(x_{1}, \ldots, x_{n}\right)\right\| \leq \prod_{i=1}^{n}\left(\left\|S_{i, m(\varepsilon)}\right\|\left\|S_{i}\left(x_{i}\right)\right\|+\varepsilon\left\|x_{i}\right\|\right) \\
\left\|T\left(x_{1}, \ldots, x_{n}\right)\right\| \leq \prod_{i=1}^{n}\left(N_{i}(\varepsilon)\left\|S_{i}\left(x_{i}\right)\right\|+\varepsilon\left\|x_{i}\right\|\right) .
\end{gathered}
$$

$b) \Rightarrow a$ ) We proceed by mathematical induction on $n$. When $n=1$, the statement follows from Proposition 1. Let us assume that our statement is true for all $k \leq n-1$. Suppose that $T: X_{1} \times \cdots \times X_{n} \rightarrow X$ is a multilinear operator satisfying b). It can be easily seen, from $b)$, that $T\left(x_{\lambda_{1}}, \ldots, x_{\lambda_{n}}\right)$ tends to zero in norm, whenever $\left(x_{\lambda_{i}}\right)$ is a bounded $S^{*}\left(X_{i}, X_{i}^{*}\right)$-null net in $X_{i}$.

For each $1 \leq i \leq n$, let $\left(x_{\mu_{i}}\right)$ be a net in $B_{X_{i}}$, converging, in the $S^{*}\left(X_{i}, X_{i}^{*}\right)$-topology to an element $x_{0}^{i} \in B_{X_{i}}$. Since, for each $1 \leq i \leq n$, $x_{\mu_{i}}-x_{0}^{i}$ is a bounded $S^{*}\left(X_{i}, X_{i}^{*}\right)$-null net in $X_{i}$, it follows that

$$
\left\|T\left(x_{\mu_{1}}-x_{0}^{1}, \ldots, x_{\mu_{n}}-x_{0}^{n}\right)\right\| \rightarrow 0 .
$$

Then we deduce that

$$
\begin{gathered}
\text { (1) } T\left(x_{\mu_{1}}, \ldots, x_{\mu_{n}}\right)-T\left(x_{\mu_{1}}-x_{0}^{1}, \ldots, x_{\mu_{n}}-x_{0}^{n}\right)=+T\left(x_{0}^{1}, x_{\mu_{2}}, \ldots, x_{\mu_{n}}\right) \\
+T\left(x_{\mu_{1}}, x_{0}^{2}, x_{\mu_{3}}, \ldots, x_{\mu_{n}}\right)+\ldots+T\left(x_{\mu_{1}}, \ldots, x_{\mu_{n-2}}, x_{0}^{n-1}, x_{\mu_{n}}\right) \\
-T\left(x_{0}^{1}, x_{0}^{2}, x_{\mu_{3}}, \ldots, x_{\mu_{n}}\right)-\ldots-T\left(x_{\mu_{1}}, \ldots, x_{\mu_{n-2}}, x_{0}^{n-1}, x_{0}^{n}\right)-\ldots \\
-(-1)^{n-1} T\left(x_{\mu_{1}}, x_{0}^{2}, \ldots, x_{0}^{n}\right)-\ldots-(-1)^{n-1} T\left(x_{0}^{1}, \ldots, x_{\mu_{n-1}}, x_{0}^{n}\right) \\
-(-1)^{n-1} T\left(x_{0}^{1}, \ldots, x_{0}^{n-1}, x_{\mu_{n}}\right)-(-1)^{n} T\left(x_{0}^{1}, \ldots, x_{0}^{n}\right)
\end{gathered}
$$

tends to zero in norm.

Let $1 \leq j \leq n-1$. We observe that whenever, in the operator $T$ we fix $j$ variables $x_{0}^{i_{1}}, \ldots, x_{0}^{i_{j}}$, then we get an $(n-j)$-multilinear operator which also satisfies statement $b)$. So, by the induction hypothesis, the latter is an $(n-$ j)-multilinear operator which is jointly Strong*-to-norm continuous on the cartesian product of the corresponding closed unit balls. This implies that every summand in the right hand side of equality (1) tends to $T\left(x_{0}^{1}, \ldots, x_{0}^{n}\right)$ in norm, which implies that $T\left(x_{\mu_{1}}, \ldots, x_{\mu_{n}}\right)$ tends to $T\left(x_{0}^{1}, \ldots, x_{0}^{n}\right)$ in norm. Thus $T$ is jointly Strong*-to-norm continuous on the cartesian product of the closed unit balls. 
The next corollary follows now from the above result together with Grothendieck's inequality for $\mathrm{C}^{*}$-algebras $[10,17]$. We recall that for each positive functional $\phi$ in the dual space of a $\mathrm{C}^{*}$-algebra $A,\|\cdot\|_{\phi}$ denotes the seminorm on $A$ defined by $\|x\|_{\phi}^{2}=2^{-1} \phi\left(x x^{*}+x^{*} x\right)$.

Corollary 5. Let $A_{1}, \ldots, A_{n}$ be $C^{*}$-algebras, $X$ a Banach space and

$$
T: A_{1} \times \cdots \times A_{n} \rightarrow X
$$

a multilinear operator. Then the following are equivalent:

a) $\left.T\right|_{B_{A_{1}} \times \ldots \times B_{A_{n}}}: B_{A_{1}} \times \ldots \times B_{A_{n}} \rightarrow X$ is jointly Strong*-to-norm continuous.

b) There exist mappings $N_{i}:(0,+\infty) \rightarrow(0,+\infty)$ (depending only on $T)$ and positive norm-one functionals $\phi_{1} \in A_{1}^{*}, \ldots, \phi_{n} \in A_{n}^{*}$ satisfying that

$$
\left\|T\left(x_{1}, \ldots, x_{n}\right)\right\| \leq \prod_{i=1}^{n}\left(N_{i}(\varepsilon)\left\|x_{i}\right\|_{\phi_{i}}+\varepsilon\left\|x_{i}\right\|\right),
$$

for each $x_{i}$ in $A_{i}$ and $\varepsilon>0$.

Corollary 6. Let $A$ be $C^{*}$-algebra and $P$ an element in $\mathcal{P}^{n}(A)$. Then the following are equivalent:

a) $\left.P\right|_{B_{A}}$ is $S^{*}\left(A, A^{*}\right)$-to-norm continuous.

b) There exists a mapping $N:(0,+\infty) \rightarrow(0,+\infty)$, (depending only on $P$ ) and positive norm-one functionals $\phi_{1}, \ldots, \phi_{n} \in A^{*}$ satisfying that

$$
|P(x)| \leq \prod_{i=1}^{n}\left(N(\varepsilon)\|x\|_{\phi_{i}}+\varepsilon\right),
$$

for each $x$ in $B_{A}$ and $\varepsilon>0$.

Corollary 7. Let $A$ be $C^{*}$-algebra and $P$ an element in $\mathcal{P}^{n}(A)$. Then the following are equivalent:

a) $\left.P\right|_{B_{A^{* *}}}$ is $S^{*}\left(A^{* *}, A^{*}\right)$-to-norm continuous.

b) There exists a mapping $N:(0,+\infty) \rightarrow(0,+\infty)$, (depending only on $P$ ) and positive norm-one functionals $\phi_{1}, \ldots, \phi_{n} \in A^{*}$ satisfying that

$$
\left|P^{* *}(x)\right| \leq \prod_{i=1}^{n}\left(N(\varepsilon)\|x\|_{\phi_{i}}+\varepsilon\right),
$$

for each $x$ in $B_{A^{* *}}$ and $\varepsilon>0$.

Corollary 8. Let $P \in \mathcal{P}^{n}(A)$ be an n-homogeneous polynomial whose restriction to the closed unit ball of $A$ is $S^{*}\left(A, A^{*}\right)$-to-norm continuous. Then the Aron-Berner extension $P^{* *}$ is $S^{*}\left(A^{* *}, A^{*}\right)$-to-norm continuous on $B_{A^{* *}}$.

Proof. Let $T: A \times \ldots \times A \rightarrow \mathbb{C}$ be the symmetric $n$-linear form satisfying that $P(x)=T(x, \ldots, x)$. By hypothesis, Lemma 2 and Corollary 5 , there exists 
a mapping $N:(0,+\infty) \rightarrow(0,+\infty)$, (depending only on $P$ ) and positive norm-one functionals $\phi_{1}, \ldots, \phi_{n} \in A^{*}$ satisfying that

$$
\left|T\left(x_{1}, \ldots, x_{n}\right)\right| \leq \prod_{i=1}^{n}\left(N(\varepsilon)\left\|x_{i}\right\|_{\phi_{i}}+\varepsilon\left\|x_{i}\right\|\right),
$$

for each $x_{i}$ in $A$ and $\varepsilon>0$. Let $z \in A^{* *},\|z\| \leq 1$. Since the $S^{*}\left(A^{* *}, A^{*}\right)$ topology is compatible with the duality $\left(A^{* *}, A^{*}\right)$, by Alaoglu's Theorem, there exists a net $\left(x_{\lambda}\right)$ in $B_{A}$ converging in the $S^{*}\left(A^{* *}, A^{*}\right)$ topology to $z$.

Since $T^{* *}$ is separately weak*-continuous and $x_{\lambda}$ tends to $z$ in the weak* topology, we deduce that

$$
\lim \left|T^{* *}\left(x_{\lambda}, x_{2}, \ldots, x_{n}\right)\right|=\left|T^{* *}\left(z, x_{2}, \ldots, x_{n}\right)\right|,
$$

for every $x_{2}, \ldots, x_{n}$. Now inequality (2) gives that

$$
\left|T^{* *}\left(z, x_{2}, \ldots, x_{n}\right)\right| \leq\left(N(\varepsilon)\|z\|_{\phi_{i}}+\varepsilon\right) \prod_{i=2}^{n}\left(N(\varepsilon)\left\|x_{i}\right\|_{\phi_{i}}+\varepsilon\right),
$$

for every $z \in B_{A^{* *}}, x_{1}, \ldots, x_{n} \in B_{A}, \varepsilon>0$.

Similarly, we have

$$
|P(z)| \leq \prod_{i=1}^{n}\left(N(\varepsilon)\|z\|_{\phi_{i}}+\varepsilon\right),
$$

for every $\varepsilon>0$ and $z \in B_{A^{* *}}$, which, by Corollary 7 shows that $P^{* *}$ is $S^{*}\left(A^{* *}, A^{*}\right)$-to-norm continuous on $B_{A^{* *}}$.

\section{MAin Result}

Let $A$ be a $\mathrm{C}^{*}$-algebra and let $X$ be a Banach space. Let $\mathcal{P}_{o}^{n}(A, X)$ denote the space of all orthogonally additive elements in $\mathcal{P}^{n}(A, X)$. When $X$ is the scalar field we just write $\mathcal{P}_{o}^{n}(A)$

A natural example of an orthogonally additive $n$-homogeneous scalar polynomial on a $\mathrm{C}^{*}$-algebra $A$ can be built as follows: Let $\phi$ be a functional in $A^{*}$, the dual space of $A$. The law $x \mapsto P_{\phi}(x):=\phi\left(x^{n}\right)$ defines an $n$-homogeneous polynomial $P_{\phi}$ on $A$. Since, for each pair of mutually orthogonal elements $x, y \in A$, we have $(x+y)^{n}=x^{n}+y^{n}$, it follows that $P_{\phi}$ is orthogonally additive.

For each $\phi$ in $A^{*}$, the polynomial $P_{\phi}$ defined as above has another interesting properties that should be also considered. $P_{\phi}$ admits a natural extension to $A^{* *}$ that will be denoted by $P_{\phi}^{* *}: A^{* *} \rightarrow \mathbb{C}, P_{\phi}^{* *}(z)=\phi\left(z^{n}\right)$. The functional $\phi$ is in the predual of $A^{* *}$ and hence $\phi$ is Strong*-to-norm continuous (see [19, Corollary 1.8.10]). Since the product of $A^{* *}$ is jointly $S^{*}\left(A^{* *}, A^{*}\right)$ continuous on bounded sets (compare [19, Proposition 1.8.12]), it follows that

$$
\left.P_{\phi}^{* *}\right|_{B_{A^{* *}}}: B_{A^{* *}} \rightarrow \mathbb{C}
$$

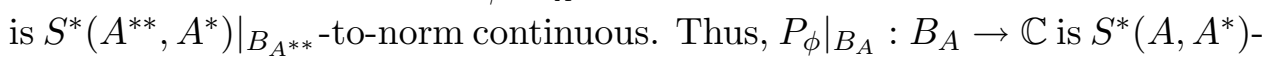
to-norm continuous. 
For each $\phi \in A^{*}$, let $T_{\phi}$ be the multilinear operator defined as follows:

$$
\begin{gathered}
T_{\phi}: A \times \ldots \times A \rightarrow \mathbb{C} \\
T_{\phi}\left(x_{1}, \ldots, x_{n}\right)=\phi\left(x_{1} x_{2} \ldots x_{n}\right) .
\end{gathered}
$$

Similar arguments to those given in the above paragraph show that $T_{\phi}$ is $S^{*}\left(A, A^{*}\right)$-to-norm continuous when restricted to the cartesian product of the closed unit ball of $A$.

Let $\mathcal{P}_{o, S^{*}}^{n}(A)$ be the space of all elements in $\mathcal{P}_{o}^{n}(A)$ whose restrictions to the closed unit ball of $A$ are $S^{*}\left(A, A^{*}\right)$-to-norm continuous.

Lemma 9. Let $A$ be a $C^{*}$-algebra and $n \in \mathbb{N}$. The mapping

$$
\Phi: A^{*} \rightarrow \mathcal{P}_{o, S^{*}}^{n}(A)
$$

defined by $\Phi(\phi)=P_{\phi}$ is an injective linear operator.

Proof. The mapping is clearly linear and bounded, with $\|\Phi\|=1$. Suppose that $P_{\phi}=P_{\varphi}$ for suitable $\phi, \varphi$ in $A^{*}$. Let $a$ be a positive element in $A$. Then there exists a positive element $x$ in $A$ such that $x^{n}=a$. Therefore $\phi(a)=P_{\phi}(x)=P_{\varphi}(x)=\varphi(a)$. Since every element $b$ in $A$ can be written as a linear combination of positive elements in $A$, we have $\phi=\varphi$.

Remark 10. The notion of orthogonality can also be defined in a natural way in a Banach lattice. For $C(K)$ spaces both notions of orthogonality (in the $C^{*}$-algebra sense and in the lattice sense) coincide. The results in our paper are inspired on the results in [16], which deal with the $C(K)$ case. The answer for $C(K)$ spaces was also proved independently in [2], where the authors also study the problem in the setting of lattices.

Let $A$ be a $\mathrm{C}^{*}$-algebra. Following [12], we say that a continuous function $f: \mathbb{R} \rightarrow \mathbb{R}$ is strongly continuous if for each $S^{*}\left(A, A^{*}\right)$-convergent net $\left(x_{\lambda}\right)$ in $A_{s a}$ with limit $x$ the net $\left(f\left(x_{\lambda}\right)\right)$ is $S^{*}\left(A, A^{*}\right)$-convergent to $\mathrm{f}(\mathrm{x})$.

Remark 11. Let $A$ be a $\mathrm{C}^{*}$-algebra. Throughout the paper $A_{s a}$ will stand for the self-adjoint part of $A$. For each element $x \in A_{s a}$ nd each $q>0$, we define $x^{[q]}=\left(x^{+}\right)^{q}-\left(x^{-}\right)^{q}$, where $x^{+}$and $x^{-}$are the positive and negative part of $x$, respectively (compare $[12,1.1 .10]$ ).

For each $n \in \mathbb{N}$ and $\psi \in A_{s a}^{*}$ the restriction of $P_{[\psi]}(x)\left(:=\psi\left(x^{[n]}\right)\right)$ to the closed unit ball of $A_{s a}$ is Strong*-to-norm continuous (although $P_{[\psi]}$ is not a polynomial if $n$ is even). Let $\left(x_{\lambda}\right)$ be a $S^{*}\left(A, A^{*}\right)$ convergent net in $B_{A_{s a}}$ with limit $x$.

Let us denote $g, h: \mathbb{R} \rightarrow \mathbb{R}$ the continuous functions defined by

$$
g(t)=\left\{\begin{array}{ll}
t, & \text { if } t \geq 0 \\
0, & \text { if } t<0
\end{array}, \text { and } h(t)= \begin{cases}t, & \text { if } t \leq 0 \\
0, & \text { if } t>0\end{cases}\right.
$$

It follows from [12, Proposition 2.3.2] that $g$ and $h$ are strongly continuous. Therefore $x_{\lambda}^{+}=g\left(x_{\lambda}\right)$ converges in the $S^{*}\left(A, A^{*}\right)$ topology to $x^{+}=g(x)$ and hence, by the joint Strong*-continuity of the product on $B_{A}$, we deduce 
that $\left(x_{\lambda}^{+}\right)^{n} \rightarrow\left(x^{+}\right)^{n}$, with respect to the $S^{*}\left(A, A^{*}\right)$ topology. Similarly, $\left(x_{\lambda}^{-}\right)^{n}=\left(h\left(x_{\lambda}\right)^{n}\right)$ converges to $\left(x^{-}\right)^{n}$ in the $S^{*}\left(A, A^{*}\right)$ topology. Thus $\left(x_{\lambda}^{[n]}\right)$ converges to $x^{[n]}$ in the $S^{*}\left(A, A^{*}\right)$ topology.

Now, by the Strong*-continuity of $\psi$, we deduce that $P_{[\psi]}\left(x_{\lambda}\right)=\psi\left(x_{\lambda}^{[n]}\right)$ tends to $\psi\left(x^{[n]}\right)=P_{[\psi]}(x)$ in norm.

Proposition 12. Let $M$ be a positive number and let $L$ be a locally compact subset of $(0, M]$ such that $L \cup\{0\}$ is compact. Let $C_{0}(L)$ denote the $C^{*}$ algebra of all complex continuous functions on $L \cup\{0\}$ vanishing at 0 . The law

$$
\phi \mapsto P_{\phi}
$$

defines a linear bijection from $C_{0}(L)^{*}$ onto $\mathcal{P}_{o}^{n}\left(C_{0}(L)\right)$. A posteriori, we get that $\mathcal{P}_{o}^{n}\left(C_{0}(L)\right)=\mathcal{P}_{o, S^{*}}^{n}\left(C_{0}(L)\right)$

Proof. The proof is the same, with the obvious modifications, as in the $C(K)$ case $([16])$.

Let $A$ be a $\mathrm{C}^{*}$-algebra. A mapping $\nu: A_{s a} \rightarrow \mathbb{R}$ is said to be a local quasi-linear functional if $\nu$ is bounded on the closed unit ball of $A_{s a}$ and for each $x \in A_{s a}, \nu$ is linear on the smallest norm closed subalgebra of $A$ containing $x$.

The solution of the Mackey-Gleason problem for von Neumann algebras obtained by L. Bunce and J. D. M. Wright in $[4,5]$ is one of the fundamental contributions to the theory of von Neumann algebras. In the just quoted papers, the authors show that for every von Neumann algebra $A$ having no direct summand of Type $I_{2}$, and for every bounded and orthogonally additive function $m$ defined on the lattice of projections of $A, m$ has a unique extension to a bounded linear functional on $A$. As a consequence, every quasi-linear functional on a von Neumann algebra $A$, where $A$ has no direct summands of Type $I_{2}$, is linear.

Let $A$ be a $\mathrm{C}^{*}$-algebra. We recall that a scalar function $\mu: A_{s a} \rightarrow \mathbb{R}$ is said to be uniformly continuous on $B_{A_{s a}}$ with respect to the $S^{*}\left(A, A^{*}\right)$-topology if for every $\varepsilon>0$ there exists an $S^{*}\left(A, A^{*}\right)$-open symmetric neighborhood of $0, V$, satisfying that for each $x, y \in B_{A_{s a}}$ with $x-y \in V$, we have $|\mu(x)-\mu(y)|<\varepsilon$.

The following result derives from [6, Theorems 3.1 and 3.3].

Proposition 13. Let $A$ be a $C^{*}$-algebra which has no quotient isomorphic to $M_{2}(\mathbb{C})$. Let $\nu: A_{s a} \rightarrow \mathbb{R}$ be a local quasi-linear mapping, which is uniformly continuous on $B_{A_{s a}}$, when the latter is equipped with the $S^{*}\left(A, A^{*}\right) \equiv$ $\left.S^{*}\left(A^{* *}, A^{*}\right)\right|_{A}$ topology. Then $\nu$ is linear.

Proof. By [6, Lemma 2.2], $A^{* *}$ has no direct summand of type $I_{2}$. Let $\pi$ : $A \rightarrow L(H)$ be the universal representation of $A$ (see [19, Definition 1.16.5]). 
By [12, Theorem 3.7.7] and [19, Proposition 1.16.2], $\pi$ extends to a weak*continuous representation $\widetilde{\pi}: A^{* *} \rightarrow L(H)$ satisfying that $\widetilde{\pi}\left(A^{* *}\right)$ is weak*closed in $L(H)$ and $\widetilde{\pi}: A^{* *} \rightarrow \widetilde{\pi}\left(A^{* *}\right)$ is a weak*-continuous isomorphism. In particular $\widetilde{\pi}$ is $S^{*}\left(A^{* *}, A^{*}\right)$-to- $S^{*}\left(\widetilde{\pi}\left(A^{* *}\right), \widetilde{\pi}\left(A^{* *}\right)_{*}\right)$ continuous (see [15, Remark 2.11] or [18, Corollary 3 and Remark 3]).

By [3, Corollary] and [18, Remark 3], it follows that

$$
\left.S^{*}\left(L(H), L(H)_{*}\right)\right|_{\tilde{\pi}\left(A^{* *}\right)}=S^{*}\left(\widetilde{\pi}\left(A^{* *}\right), \tilde{\pi}\left(A^{* *}\right)_{*}\right) .
$$

Since we also have $S^{*}\left(A, A^{*}\right)=\left.S^{*}\left(A^{* *}, A^{*}\right)\right|_{A}$ we deduce that $[\nu]: \pi\left(A_{s a}\right) \rightarrow \mathbb{R},[\nu](\pi(x))=x,\left(x \in A_{s a}\right)$ is a local quasi-linear functional which is uniformly continuous on the closed unit ball of $\pi\left(A_{s a}\right)$. Finally, Theorem 3.1 in [6] implies that $[\nu]$ (and hence $\nu$ ) is linear.

In the above result the hypothesis of $\nu$ being uniformly continuous on $B_{A_{s a}}$ is cannot always easily checked. In [6, Theorem 3.1], this hypothesis is needed to guarantee the existence of an extension of $\nu$ to $A_{s a}^{* *}$. In our particular setting of orthogonally additive polynomials, the existence of this extension will be assured by the Aron-Berner transpose. Similar ideas to those presented in [6, Theorem 3.1] are also enough to get the statement of following lemma. The proof is included here for completeness reasons.

Lemma 14. Let $A$ be a $C^{*}$-algebra which has no quotient isomorphic to $M_{2}(\mathbb{C})$. Let $\nu: A_{s a} \rightarrow \mathbb{R}$ be a local quasi-linear mapping satisfying the following:

a) $\nu$ is $S^{*}\left(A, A^{*}\right)$-to-norm continuous on $B_{A_{s a}}$

b) $\nu$ admits an extension $\bar{\nu}: A_{s a}^{* *} \rightarrow \mathbb{R}$ which is $S^{*}\left(A^{* *}, A^{*}\right)$-to-norm continuous on $B_{A_{s a}^{* *}}$.

c) $\sup \left\{|\bar{\nu}(p)|: p\right.$ is a projection in $\left.A^{* *}\right\}<\infty$.

Then $\bar{\nu}$ is local quasi-linear and $\nu$ and $\bar{\nu}$ are linear.

Proof. Let us observe that every norm convergent sequence is bounded and converges also in the Strong* topology. Thus $\nu$ and $\bar{\nu}$ are clearly norm continuous.

We shall now show that $\bar{\nu}$ is local quasi-linear. To this end, let $z$ be a nonzero element in $A_{s a}^{* *}$. We may assume that $\|z\|=1$. Since the $S^{*}\left(A^{* *}, A^{*}\right)$ topology is compatible with the duality $\left(A^{* *}, A^{*}\right)$, by Alaoglu's Theorem, there exists a net $\left(x_{\lambda}\right)$ in $B_{A}$ converging in the $S^{*}\left(A^{* *}, A^{*}\right)$ topology to $z$.

Since $\bar{\nu}$ is an extension of $\nu$ and $\bar{\nu}$ is $S^{*}\left(A^{* *}, A^{*}\right)$-to-norm continuous on

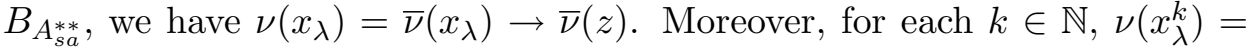
$\bar{\nu}\left(x_{\lambda}^{k}\right) \rightarrow \bar{\nu}\left(z^{k}\right)$. This implies that whenever $\Upsilon_{1}$ and $\Upsilon_{2}$ are real polynomials with zero constant term, we have

$$
\nu\left(\Upsilon_{1}\left(a_{\lambda}\right)\right)+\nu\left(\Upsilon_{2}\left(a_{\lambda}\right)\right)=\nu\left(\Upsilon_{1}\left(a_{\lambda}\right)+\Upsilon_{2}\left(a_{\lambda}\right)\right),
$$


by the local quasi-linearity of $\nu$. And, by the joint $S^{*}\left(A^{* *}, A^{*}\right)$-continuity of the product on bounded sets we deduce $\Upsilon_{i}\left(a_{\lambda}\right) \rightarrow \Upsilon_{i}(z)$ in the $S^{*}\left(A^{* *}, A^{*}\right)$ topology $(i=1,2)$. The corresponding continuity of $\bar{\nu}$ gives

$$
\bar{\nu}\left(\Upsilon_{1}(z)\right)+\bar{\nu}\left(\Upsilon_{2}(z)\right)=\bar{\nu}\left(\Upsilon_{1}(z)+\Upsilon_{2}(z)\right)
$$

Standard norm-continuity arguments allow us to conclude that $\bar{\nu}$ is linear in the norm closed subalgebra of $A_{s a}^{* *}$ generated by $z$. This shows that $\bar{\nu}$ is local quasi-linear.

Let $p_{1}, p_{2}, \ldots, p_{k}$ be orthogonal projections in $A_{s a}^{* *}$ and denote $z=p_{1}+$ $\frac{1}{2} p_{2}+\ldots+\frac{1}{2^{k-1}} p_{k}+\frac{1}{2^{k}}\left(1-p_{1}-p_{2}-\ldots-p_{k}\right)$. The sequence $\left(z^{m}\right)_{m}$ converges in norm to $p_{1}$. This implies that $p_{1}$ lies is the norm closed subalgebra of $A_{s a}^{* *}$ generated by $z$. The sequence $\left(\left(2 z-2 p_{1}\right)^{m}\right)_{m}$ converges in norm to $p_{2}$. This argument can be repeated to show that $p_{1}, p_{2}, \ldots, p_{k}$ all lie in the norm closed subalgebra generated by $z$. The local quasi-linearity of $\bar{\nu}$ gives that $\bar{\nu}$ is a finitely additive measure on the projections of $A^{* *}$.

Let us observe that, by [6, Lemma 2.2], $A^{* *}$ has no direct summands of type $M_{2}(\mathbb{C})$. Now the Bunce-Wright-Mackey-Gleason Theorem ([4, Theorem B] or [5, Theorem B]) guarantees that $\bar{\nu}$ extends to a bounded linear functional on $A_{s a}^{* *}$. Finally, by local quasi-linearity and spectral theory we conclude that $\nu$ and $\bar{\nu}$ are linear.

We will need the following technical lemma.

Lemma 15. Let $A$ be a $C^{*}$-algebra, $n \in \mathbb{N}$ and $P, Q \in \mathcal{P}^{n}(A)$. Suppose that $P(a)=Q(a)$, for every $a \in A_{s a}$. Then $P(x)=Q(x)$, for every $x \in A$.

Proof. We shall proceed by mathematical induction on $n$. When $n=1$ the statement follows immediately. Let us then assume that our thesis holds for $n-1$. Assume $P, Q \in \mathcal{P}^{n}(A)$ are such that $P(a)=Q(a)$, for every $a \in A_{\text {sa }}$. Let $T, S: A \times \ldots \times A \rightarrow \mathbb{C}$ be the symmetric $n$-linear forms satisfying that $P(x)=T(x, \ldots, x)$ and $Q(x)=S(x, \ldots, x)$ for every $x \in A$. By hypothesis we know that $T(a, \ldots, a)=S(a, \ldots, a)$, for every $a \in A_{s a}$, and hence, by the polarization formula $[11, \mathrm{p} .6], T\left(a_{1}, \ldots, a_{n}\right)=S\left(a_{1}, \ldots, a_{n}\right)$ for every $a_{1}, \ldots, a_{n} \in A_{s a}$.

Fixed $a \in A_{s a}$, we define the symmetric $n-1$ linear forms

$$
T_{a}, S_{a}: A \times \ldots \times A \rightarrow \mathbb{C}
$$

as

$$
T_{a}\left(x_{1}, \ldots, x_{n-1}\right):=T\left(a, x_{1}, \ldots, x_{n-1}\right)
$$

and

$$
S_{a}\left(x_{1}, \ldots, x_{n-1}\right):=S\left(a, x_{1}, \ldots, x_{n-1}\right) .
$$

We take their associated $(n-1)$-homogeneous polynomials $P_{a}, Q_{a}: A \rightarrow \mathbb{C}$ given by $P_{a}(x)=T_{a}(x, \ldots, x)$ and $Q_{a}(x)=S_{a}(x, \ldots, x)$, for every $x \in$ $A$. We have seen above that, for each $b \in A_{s a}, P_{a}(b)=Q_{a}(b)$ so, by the induction hypothesis, we conclude that $P_{a}(x)=Q_{a}(x)$ for every $x \in A$. Then we have $T(a, x \ldots, x)=S(a, x \ldots, x)$, for every $a \in A_{s a}$ and $x \in A$. 
Now, using the linearity of $T$ and $S$ it follows that $P(x)=T(x, \ldots, x)=$ $S(x, \ldots, x)=Q(x)$, for every $x \in A$.

We can now prove our main result:

Theorem 16. Let $A$ be a $C^{*}$-algebra which has no quotient isomorphic to $M_{2}(\mathbb{C})$. For each $P$ in $\mathcal{P}_{o, S^{*}}^{n}(A)$ there exists $\varphi$ in $A^{*}$ satisfying $P(x)=\varphi\left(x^{n}\right)$, for each element $x$ in $A$.

Proof. Suppose that $P \in \mathcal{P}_{o, S^{*}}^{n}(A)$. Let $T: A \times \ldots \times A \rightarrow \mathbb{C}$ be the symmetric $n$-linear form satisfying that $P(x)=T(x, \ldots, x)$ and let $T^{* *}: A^{* *} \times \ldots \times$ $A^{* *} \rightarrow \mathbb{C}$ denote the corresponding Aron-Berner extension of T. $P^{* *}$ will stand for the polynomial on $A^{* *}$ defined by $P^{* *}(z)=T^{* *}(z, \ldots, z),(z$ in $\left.A^{* *}\right)$.

Let us define two mappings $\mu: A \rightarrow \mathbb{C}$ and $\bar{\mu}: A^{* *} \rightarrow \mathbb{C}$ given by the laws

$$
\begin{gathered}
\mu(a)=\Re \mathrm{e} P\left(a^{1 / n}\right), \text { for each } a \in A^{+}, \\
\mu(b)=\mu\left(b^{+}\right)-\mu\left(b^{-}\right), \text {for each } b \in A_{s a}, \\
\mu(b+i c)=\mu(b)+i \mu(c), \quad\left(\forall b, c \in A_{s a}\right),
\end{gathered}
$$

and

$$
\begin{gathered}
\bar{\mu}(a)=\Re \mathrm{e} P^{* *}\left(a^{1 / n}\right), \text { for each } a \in\left(A^{* *}\right)^{+}, \\
\bar{\mu}(b)=\bar{\mu}\left(b^{+}\right)-\bar{\mu}\left(b^{-}\right), \text {for each } b \in A_{s a}^{* *} \\
\bar{\mu}(b+i c)=\bar{\mu}(b)+i \bar{\mu}(c), \quad\left(\forall b, c \in A_{s a}^{* *}\right)
\end{gathered}
$$

It is clear that $\sup \left\{|\bar{\mu}(p)|: p\right.$ is a projection in $\left.A^{* *}\right\} \leq\left\|P^{* *}\right\|<\infty$.

Since $P$ is $S^{*}\left(A, A^{*}\right)$-to-norm continuous on the closed unit ball of $A$, we deduce from Corollary 8 and Lemma 2 that $T$ (respectively, $T^{* *}$ ) is jointly $S^{*}\left(A, A^{*}\right)$-continuous on $B_{A} \times \ldots \times B_{A}$ (respectively, jointly $S^{*}\left(A^{* *}, A^{*}\right)$ continuous on $\left.B_{A^{* *}} \times \ldots \times B_{A^{* *}}\right)$.

As we have seen in Remark 11, the function $f: \mathbb{R} \rightarrow \mathbb{R}$ defined by

$$
f(t)=\left\{\begin{array}{ll}
\sqrt[n]{t}, & \text { if } t \geq 0 \\
-\sqrt[n]{-t}, & \text { if } t<0
\end{array},\right.
$$

is strongly continuous (compare [12, Proposition 2.3.2]). This fact together with the above paragraph imply that $\mu$ (respectively, $\bar{\mu})$ is $S^{*}\left(A, A^{*}\right)$-tonorm continuous on $B_{A}$ (respectively, $S^{*}\left(A^{* *}, A^{*}\right)$-to-norm continuous on $\left.B_{A^{* *}}\right)$. We also have $\mu(a)=\bar{\mu}(a)$, for each $a \in A$.

In order to simplify notation, we write $\nu$ for the restriction of $\mu$ to $A_{s a}$ and $\bar{\nu}$ for the restriction of $\bar{\nu}$ to $A_{s a}^{* *}$.

We claim that $\nu$ is local quasi linear. Indeed, for each $x \in A_{s a}$ let $C_{x}$ be the smallest norm closed subalgebra of $A_{s a}$ containing $x$. It is known that $C_{x}$ is isomorphically isometric to $C_{0}(L, \mathbb{R})$ for a locally compact Hausdorff space $L \subset(0,\|x\|]$ with $L \cup\{0\}$ compact.

Since $P \in \mathcal{P}_{o}^{n}(A)$ (and thus, $\Re \mathrm{e} P \in \mathcal{P}_{o}^{n}\left(A_{s a}\right)$ ) we have that $Q$, the restriction of $\Re \mathrm{e} P$ to $C_{x}$, also is orthogonally additive. Now, by Proposition 12 , 
we know that there exists a functional $\varphi_{x} \in\left(C_{x}\right)^{*}$ such that $Q(z)=\varphi_{x}\left(z^{n}\right)$ for every $z \in C_{x}$. Now, we take a positive element $a \in C_{x}$ and a positive element $y \in C_{x}$ with $y^{n}=a$. Then $\nu(a)=\nu\left(y^{n}\right)=Q(y)=\varphi_{x}\left(y^{n}\right)=\varphi(a)$. This implies, immediately, that $\nu=\varphi$ on $C_{x}$. Therefore $\left.\nu\right|_{C_{x}}: C_{x} \rightarrow \mathbb{R}$ is linear, which implies that $\nu$ is local quasi-linear.

Now, Lemma 14 gives that $\nu: A_{s a} \rightarrow \mathbb{R}$ is linear. As a consequence

$$
\mu\left(x^{n}\right)=\nu\left(x^{n}\right)=\nu\left(\left(x^{+}\right)^{n}+(-1)^{n}\left(x^{-}\right)^{n}\right)=\nu\left(\left(x^{+}\right)^{n}\right)+(-1)^{n} \nu\left(\left(x^{-}\right)^{n}\right)
$$

(by definition of $\nu$ ) $=\Re \mathrm{e} P\left(x^{+}\right)+(-1)^{n} \Re \mathrm{e} P\left(x^{-}\right)=\Re \mathrm{e} P\left(x^{+}\right)+\Re \mathrm{e} P\left(-x^{-}\right)$

(by orthogonality) $=\Re \mathrm{e} P\left(x^{+}-x^{-}\right)=\Re \mathrm{e} P(x)$,

for every $x \in A_{s a}$. Similarly, we can get a linear functional $\psi: A_{s a} \rightarrow \mathbb{R}$ such that $\psi\left(x^{n}\right)=: \Im \mathrm{m} P(x)$, for every $x \in A_{s a}$. We then have a linear form $\varphi(a+i b):=\nu(a)+i \psi(b)$ in $A^{*}$ satisfying that $P(a)=\varphi\left(a^{n}\right)$, for every $a \in A_{s a}$.

Finally, let $O \in \mathcal{P}_{o, S^{*}}^{n}(A)$, be the polynomial defined by $O(x):=\varphi\left(x^{n}\right)$. Since, for each $a \in A_{s a}, O(a)=\varphi\left(a^{n}\right)=P(a)$, Lemma 15 gives the statement.

Corollary 17. Let $A$ be a $C^{*}$-algebra which has no quotient isomorphic to $M_{2}(\mathbb{C})$ and let $n \in \mathbb{N}$. The operator

$$
\begin{gathered}
\Phi: A^{*} \rightarrow \mathcal{P}_{o, S^{*}}^{n}(A) \\
\Phi(\varphi)=P_{\varphi}
\end{gathered}
$$

is a bicontinuous linear bijection from $A^{*}$ onto $\mathcal{P}_{o, S^{*}}^{n}(A)$. Moreover, for each $\phi \in A_{s a}^{*},\|\phi\|=\left\|P_{\phi}\right\|$ while $\|\phi\| \geq\left\|P_{\phi}\right\| \geq 2\|\phi\|$, for all $\phi \in A^{*}$.

Let $A$ be a $\mathrm{C}^{*}$-algebra. By the bilinear Grothendieck's inequality for $\mathrm{C}^{*}$ algebras (see $[17,10]$ ), we clearly have $\mathcal{P}_{S^{*}}^{2}(A)=\mathcal{P}^{2}(A)$. The failure of a trilinear Grothendieck's inequality implies that $\mathcal{P}_{S^{*}}^{n}(A) \neq \mathcal{P}^{n}(A)$, for $n \geq 3$. It seem natural to ask whether $\mathcal{P}_{o, S^{*}}^{n}(A)=\mathcal{P}_{o}^{n}(A)$.

Problem 18. Let $A$ be a $C^{*}$-algebra. Let $P$ be an orthogonally additive $n$-homogeneous polynomial on $A$. Is $P$ automatically $S^{*}\left(A, A^{*}\right)$-to-norm continuous on bounded sets?

\section{Vector valued polynomials}

We consider now the space $\mathcal{P}_{o, S^{*}}^{n}(A, X)$ be the space of all elements in $\mathcal{P}_{o}^{n}(A, X)$ whose restrictions to the closed unit ball of $A$ are $S^{*}\left(A, A^{*}\right)$-tonorm continuous.

As in the scalar case, it is easy to see that, for every $m \in \mathcal{L}(A, X)$ we can define a polynomial $P_{m} \in \mathcal{P}_{o, S^{*}}^{n}(A, X)$ by

$$
P_{m}(a)=m\left(a^{n}\right) \text {. }
$$

We have 
Corollary 19. Let $A$ be a $C^{*}$-algebra which has no quotient isomorphic to $M_{2}(\mathbb{C})$. For each $P$ in $\mathcal{P}_{o, S^{*}}^{n}(A, X)$ there exists $m \in \mathcal{L}(A, X)$ satisfying $P(a)=m\left(a^{n}\right)$, for each element a in $A$. Therefore, the operator

$$
\begin{gathered}
\Phi: \mathcal{L}(A, X) \longrightarrow \mathcal{P}_{o, S^{*}}^{n}(A, X) \\
\Phi(m)=P_{m}
\end{gathered}
$$

is a bicontinuous linear bijection from $\mathcal{L}(A, X)$ onto $\mathcal{P}_{o, S^{*}}^{n}(A, X)$.

Proof. Everything is either immediate or similar to the scalar case except (maybe) for the surjectivity. We prove this. Let $P$ be as in the hypothesis. In that case, for every $x^{*} \in X^{*}, x^{*} \circ P \in \mathcal{P}_{o, S^{*}}^{n}(A)$. Hence, Theorem 16 guarantees the existence of an element $m_{x^{*}} \in A^{*}$ such that, for every $a \in A$,

$$
x^{*} \circ P(a)=m_{x^{*}}\left(a^{n}\right) .
$$

So, we define a mapping

$$
m: A \longrightarrow X^{* *}
$$

by

We need to check that

$$
\left\langle m(a), x^{*}\right\rangle=m_{x^{*}}(a) .
$$

- $m(a)$ is indeed in $X^{* *}$. First we check that $m(a): X^{*} \longrightarrow \mathbb{K}$ is linear. We first consider the case when $a \geq 0$. Then

$$
\begin{gathered}
\left\langle m(a), \alpha x^{*}+\beta y^{*}\right\rangle=m_{\alpha x^{*}+\beta y^{*}}(a)=\left(\left(\alpha x^{*}+\beta y^{*}\right) \circ P\right)\left(a^{\frac{1}{n}}\right)= \\
=\alpha\left(x^{*} \circ P\right)\left(a^{\frac{1}{n}}\right)+\beta\left(y^{*} \circ P\right)\left(a^{\frac{1}{n}}\right)=\alpha m_{x^{*}}(a)+\beta m_{y^{*}}(a)= \\
=\alpha\left\langle m(a), x^{*}\right\rangle+\beta\left\langle m(a), y^{*}\right\rangle
\end{gathered}
$$

Using this we can prove that $m(a): X^{*} \longrightarrow \mathbb{K}$ is linear for a general $a \in A$ using the decomposition of $a$ as linear combination of positive elements and standard reasonings

We now have to check that $m(a): X^{*} \longrightarrow \mathbb{K}$ is bounded, but this is easier.

- $m$ is linear: for every $x^{*} \in X^{*}$,

$$
\begin{aligned}
\left\langle m(\alpha a+\beta b), x^{*}\right\rangle & =m_{x^{*}}(\alpha a+\beta b)= \\
=\alpha m_{x^{*}}(a)+\beta m_{x^{*}}(b) & =\left\langle\alpha m(a)+\beta m(b), x^{*}\right\rangle
\end{aligned}
$$

- $m$ is bounded: Let $a \in B_{A}$. We first assume that $a$ is positive. Then

$$
\|m(a)\|=\sup _{x^{*} \in B_{X^{*}}}\left|\left\langle m(a), x^{*}\right\rangle\right|=\sup _{x^{*} \in B_{X^{*}}}\left|x^{*} \circ P\left(a^{\frac{1}{n}}\right)\right|=\left\|P\left(a^{\frac{1}{n}}\right)\right\| \leq\|P\| .
$$

Again, we can remove now the condition that $a$ be positive.

- For every $a \in A, m\left(a^{n}\right)=P(a)$ : For every $x^{*} \in X^{*}$ we have

$$
\left\langle m\left(a^{n}\right), x^{*}\right\rangle=m_{x^{*}}\left(a^{n}\right)=x^{*} \circ P(a)
$$

and the desired inequality follows. A posteriori, we have that for every positive $a \in A, m(a) \in X$, and it follows that $m$ is actually $X$-valued. 


\section{REFERENCES}

[1] Aron, R. M. and Berner P. D., A Hahn-Banach extension theorem for analytic mappings, Bull. Soc. Math. France 106, 3-24 (1978).

[2] Benyamini, Y., Lassalle and Llavona, J. G., Homogeneous orhtogonally additive polynomials on Banach lattices, Bull. London Math. Soc. 38, 459-469 (2006).

[3] Bunce, L. J., Norm preserving extensions in JBW*-triple preduals, Quart. J. Math. Oxford 52, no. 2, 133-136 (2001).

[4] Bunce, L. J. and Wright, J. D. M., The Mackey-Gleason problem, Bull. Amer. Math. Soc. 26, no. 2, 288-293 (1992).

[5] Bunce, L. J. and Wright, J. D. M., The Mackey-Gleason problem for vector measures on projections in von Neumann algebras, J. London Math. Soc. (2) 49, no. 1, 133-149 (1994).

[6] Bunce, L. and Wright, J. D. M., The quasi-linear problem for $\mathrm{C}^{*}$-algebras, Pacific J. Math. 172, No. 1, 41-47 (1996).

[7] Drewnowski, L. and Orlicz, W., Continuity and representation of orthogonally additive functionals, Bull. Acad. Polon. Sci. Ser. Sci. Math. Astronom. Phys. 17, 647û-653 (1969).

[8] Friedman, N. and Katz M., Additive functionals of Lp spaces, Canad. J. Math. 18, $1264 \hat{u} 1271$ (1966).

[9] Friedman, N. and Katz M., A representation theorem for additive functionals, Arch. Rational Mech. Anal. 21 49û-57 (1966).

[10] Haagerup, U., The Grothendieck inequality for bilinear forms on $\mathrm{C}^{*}$-algebras, Avd. Math. 56, 93-116 (1985).

[11] J. Mújica, Complex Analysis in Banach spaces, Math. Studies No. 120. North-Holland, 1986.

[12] Pedersen, G. K., C*algebras and their automorphism groups, Academic Press, 1979.

[13] Peralta, A. M., Topological characterisation of weakly compact operators revisited, preprint 2006.

[14] Peralta, A. M., Villanueva, I., Wright, J.D.M. and Ylinen, K., Topological characterisation of weakly compact operators, to appear in J. Math. Anal. Appl.

[15] Peralta, A. M., Villanueva, I., Wright, J.D.M. and Ylinen, K., The Strong*-topology and quasi completely continuous operators on Banach spaces, preprint 2006.

[16] Pérez, D. and Villanueva, I., Orthogonally additive polynomials on spaces of continuous functions, J. Math. Anal. Appl. 306, 97-105, (2005)

[17] Pisier, G., Grothendieck's theorem for non commutative $\mathrm{C}^{*}$-algebras with an appendix on Grothendieck's constant, J. Funct. Anal. 29, 397-415 (1978).

[18] Rodríguez Palacios, Á., On the strong* topology of a JBW*-triple, Quart. J. Math. Oxford (2) 42, 99-103 (1991).

[19] Sakai, S., $C^{*}$ - and $W^{*}$-algebras, Springer-Verlag, Berlin-New York, 1971.

[20] Sundaresam, K., Geometry of spaces of polynomials on Banach lattices, in: $A p$ plied Geometry and Discrete Mathematics, in: DIMACS Ser. Discrete Math. Theoret. Comput. Sci., vol. 4, Amer. Math. Soc., Providence, RI, 1991, pp. 571 û586.

[21] M. Takesaki, Theory of operator algebras I, Springer Verlag, New York-Heidelberg, 1979.

E-mail address: carlospalazuelos@mat.ucm.es

Departamento de Análisis Matemático, Facultad de Matemáticas, UniverSidad Complutense de Madrid, Madrid 28040, Spain

E-mail address: aperalta@ugr.es 
Departamento de Análisis Matemático, Facultad de Ciencias, Universidad de Granada, 18071 Granada, Spain.

E-mail address: ignaciov@mat.ucm.es

Departamento de Análisis Matemático, Facultad de Matemáticas, UniverSidad Complutense de Madrid, Madrid 28040, Spain 